Commun. math. Phys. 22, 44-50 (1971)

(C) by Springer-Verlag 1971

\title{
Vecteurs totalisateurs d'une algèbre de von Neumann
}

\author{
J. Dixmier et O. Maréchal \\ Université de Paris VI
}

Reçu le 16 février 1971

\begin{abstract}
We prove that the set of cyclic vectors for a von Neumann algebra in a Hilbert space $H$ is a $G_{\delta}$ set, which is empty or dense. We obtain some corollaries, for instance: if $\left(\mathscr{A}_{1}, \mathscr{A}_{2}, \ldots\right)$ is a sequence of von Neumann algebras in $H$, and if each $\mathscr{A}_{n}$ has a cyclic vector and a separating vector, then there exists a vector in $H$ which is cyclic and separating for each $\mathscr{A}_{n}$. For algebras of local observables, we improve the known results connecting the infinite type of the algebras and the existence of cyclic and separating vectors.

Notations et définitions. Si $\mathscr{A}$ est une algèbre de von Neumann sur l'espace hilbertien $H$, on note $\operatorname{Tot}(\mathscr{A})(\operatorname{resp} . \operatorname{Sep}(\mathscr{A}))$ l'ensemble des vecteurs totalisateurs (resp. séparateurs) de $\mathscr{A}$. On note $\operatorname{Inv}(\mathscr{A})$ l'ensemble des éléments inversibles de $\mathscr{A}$. Si $\mathscr{A}$ est finie ainsi que son commutant, on note $C_{\mathscr{A}}$ la fonction de liaison de $\mathscr{A}$ ([4], Chap. III, $\left.\S 6\right)$. Si $M \subset H$ et $\mathscr{A} \subset \mathscr{L}(H)$, on note $[\mathscr{A} M]$ le projecteur sur le sous-espace vectoriel fermé engendré par les $A x(A \in \mathscr{A}, x \in M)$. Si $U$ et $V$ sont des isométries partielles, on dit que $U$ domine $V$ lorsque: 1$) \operatorname{Ker} U(\operatorname{Ker} V$;2) $U$ coincide avec $V$ sur le sous-espace initial de $V$.

Soit $\Omega$ l'ensemble des ouverts bornés dans l'espace de Minkowski $M=\boldsymbol{R}^{4}$. Soient $\mathcal{O}_{1}, \mathcal{O}_{2} \in \Omega$. On écrira $\mathcal{O}_{1}<\mathcal{O}_{2}$ si $\mathcal{O}_{1} \subset \mathcal{O}_{2}$ et s'il existe $\mathcal{O} \in \Omega$ tel que: 1) $\left.\mathcal{O} \subset \mathcal{O}_{2} ; 2\right) \mathcal{O}$ et $\mathcal{O}_{1}$ sont causalement disjoints.
\end{abstract}

\section{Densité des vecteurs totalisateurs}

Lemme 1. Soient $\mathscr{A}$ une algèbre de von Neumann et $U \in \mathscr{A}$ une isométrie partielle. Il existe un projecteur $G$ du centre de $\mathscr{A}$ tel que $U_{G}$ soit dominée par une isométrie de $\mathscr{A}_{G}$ et $U_{1-G}$ par une coisométrie de $\mathscr{A}_{1-G}$.

Soit $E$ (resp. $F$ ) le projecteur initial (resp. final) de $U$. Il existe ([4], Chap. III, $\S 1$, Th. 1) un projecteur $G$ du centre de $\mathscr{A}$ tel que $(1-E) G<(1-F) G$ et $(1-E)(1-G) \succ(1-F)(1-G)$. Soit $V$ une isométrie partielle de $\mathscr{A}$ de projecteur initial $(1-E) G$ et dont le projecteur final est majoré par $(1-F) G$. Alors $(U+V)_{G}$ est une isométrie de $\mathscr{A}_{G}$ qui domine $U_{G}$. De même, si $V_{1}$ est une isométrie partielle de $\mathscr{A}$ 
de projecteur final $(1-F)(1-G)$ et dont le projecteur initial est majoré par $(1-E)(1-G)$, alors $\left(U+V_{1}\right)_{1-G}$ est une coisométrie de $\mathscr{A}_{1-G}$ qui domine $U_{1-G}$.

Lemme 2. Soient $\mathscr{A}$ une algèbre de von Neumann proprement infinie et $U \in \mathscr{A}$ une isométrie. Alors $U$ est limite forte d'une suite d'unitaires de $\mathscr{A}$.

Soit $F$ le projecteur final de $U$. Soit $\left(E_{1}, E_{2}, \ldots\right)$ une suite de projecteurs deux à deux orthogonaux, équivalents à 1 , de somme 1 ([4], Chap. III, $\S 8$, Cor. $2 \mathrm{du}$ Th. 1). Soit $E_{n}^{\prime}=\sum_{i=1}^{n} E_{i}$ et soit $F_{n}^{\prime}$ le projecteur final de $U E_{n}^{\prime}$. On a $1-E_{n}^{\prime} \geqq E_{n+1} \sim 1$ donc $1-E_{n}^{\prime} \sim 1$, donc $F-F_{n}^{\prime} \sim 1$; par suite $1-F_{n}^{\prime} \geqq F-F_{n}^{\prime} \sim 1$ et finalement $1-E_{n}^{\prime} \sim 1-F_{n}^{\prime}$. Soit $V_{n} \in \mathscr{A}$ une isométrie partielle de projecteur initial $1-E_{n}^{\prime}$ et de projecteur final $1-F_{n}^{\prime}$. Alors $U E_{n}^{\prime}+V_{n}$ est un unitaire de $\mathscr{A}$. Comme $E_{n}^{\prime} \rightarrow 1$ fortement et que $U E_{n}^{\prime}+V_{n}$ coincide avec $U$ sur l'image de $E_{n}^{\prime}$, on a $U E_{n}^{\prime}+V_{n} \rightarrow U$ fortement.

Lemme 3. Soient $\mathscr{A}$ une algèbre de von Neumann proprement infinie et $U \in \mathscr{A}$ une coisométrie. Alors $U$ est limite forte d'une suite d'éléments inversibles de $\mathscr{A}$.

Soit $E$ le projecteur initial de $U$. Comme le projecteur final de $U$ est 1 , on a $E \sim 1$, donc $E$ est proprement infini. Donc il existe une suite $\left(E_{1}, E_{2}, \ldots\right)$ de projecteurs de $\mathscr{A}$, deux à deux orthogonaux, de somme $E$, équivalents à $E$ donc à 1 . Soit $E_{n}^{\prime}=\sum_{i=1}^{n} E_{i}$ et soit $F_{n}^{\prime}$ le projecteur final de $U E_{n}^{\prime}$. On a $E-E_{n}^{\prime} \geqq E_{n+1} \sim 1$ donc $1-E_{n}^{\prime} \sim 1$ et $1-F_{n}^{\prime} \sim E-E_{n}^{\prime} \sim 1$ d'où $1-E_{n}^{\prime} \sim 1-F_{n}^{\prime}$. Soit $V_{n} \in \mathscr{A}$ une isométrie partielle admettant $1-E_{n}^{\prime}$ comme projecteur initial et $1-F_{n}^{\prime}$ comme projecteur final. Alors $U E_{n}^{\prime}+(1 / n) V_{n} \in \operatorname{Inv}(\mathscr{A})$. Comme $E_{\dot{n}}^{\prime} \rightarrow E$ fortement, $U E_{n}^{\prime}+(1 / n) V_{n} \rightarrow U$ fortement.

Proposition 1. Soit $\mathscr{A}$ une algèbre de von Neumann. Tout élément de $\mathscr{A}$ est limite forte d'une suite d'éléments inversibles de $\mathscr{A}$.

(Pour la topologie normique, le résultat est très différent; cf. [5], Th. 1.)

Soit $A \in \mathscr{A}$ et soit $A=U R$ sa décomposition polaire avec $U$ partiellement isométrique et $R \geqq 0$. On a $U \in \mathscr{A}$ et $R \in \mathscr{A}$. Pour toute isométrie partielle $V$ dominant $U$, on a encore $A=V R$. D'après la théorie spectrale, $R$ est limite normique d'une suite d'hermitiens inversibles de $\mathscr{A}$; il suffit donc de montrer que, pour toute isométrie partielle $U$ de $\mathscr{A}$, il existe une isométrie partielle $V$ de $\mathscr{A}$ dominant $U$ et telle que $V$ soit limite forte d'une suite d'éléments inversibles de $\mathscr{A}$. D'autre part, puisque $\mathscr{A}$ est produit d'une algèbre finie et d'une algèbre proprement infinie, il suffit de démontrer le résultat dans le cas où $\mathscr{A}$ est soit finie, soit proprement infinie. Si $\mathscr{A}$ est finie, cela résulte du Lemme 1 et du fait que les 
isométries et les coisométries de $\mathscr{A}$ sont unitaires ([4], Chap. III, $\S 1$, Prop. 3). Si $\mathscr{A}$ est proprement infinie, cela résulte des Lemmes 1,2, 3 .

Lemme 4. Soient $H$ un espace hilbertien et $\mathscr{A}$ une algèbre de von

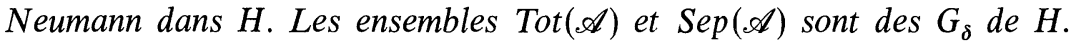

Si $\operatorname{Tot}(\mathscr{A})=\emptyset, \operatorname{Tot}(\mathscr{A})$ est un $G_{\delta}$. Supposons qu'il existe un $x_{0} \in \operatorname{Tot}(\mathscr{A})$. Pour $A \in \mathscr{A}$ et $n=1,2, \ldots$, soit $\omega(A, n)$ l'ouvert de $H$ défini par $\omega(A, n)=\left\{x \in H \mid\left\|A x-x_{0}\right\|<1 / n\right\}$. Un élément $y$ de $H$ appartient à $\operatorname{Tot}(\mathscr{A})$ si et seulement si, pour tout $n$, il existe $A \in \mathscr{A}$ tel que $y \in \omega(A, n)$. Autrement dit,

$$
\operatorname{Tot}(\mathscr{A})=\bigcap_{n \geqq 1} \bigcup_{A \in \mathscr{A}} \omega(A, n)
$$

de sorte que $\operatorname{Tot}(\mathscr{A})$ est un $G_{\delta}$. En remplaçant $\mathscr{A}$ par $\mathscr{A}^{\prime}$, on voit que $\operatorname{Sep}(\mathscr{A})$ est aussi un $G_{\delta}$.

Proposition 2. Soient $H$ un espace hilbertien et $\mathscr{A}=\mathscr{A}_{1} \times \mathscr{A}_{2}$ une algèbre de von Neumann sur $H$, l'algèbre $\mathscr{A}_{1}$ étant proprement infinie et $\mathscr{A}_{2}$ finie. Les 3 conditions suivantes sont équivalentes:

(i) $\operatorname{Tot}(\mathscr{A}) \neq \emptyset$.

(ii) $T o t(\mathscr{A})$ est un $G_{\delta}$ dense dans $H$.

(iii) $\mathscr{A}^{\prime}$ est de genre dénombrable, $\mathscr{A}_{2}^{\prime}$ est finie et $C_{\mathscr{A}_{2}} \leqq 1$.

(L'équivalence de (i) et (iii) est déjà connue, mais nous en rappelons la démonstration.)

Soit $H=H_{1} \oplus H_{2}$, l'algèbre $\mathscr{A}_{1}$ opérant dans $H_{1}$ et $\mathscr{A}_{2}$ dans $H_{2}$. On a $\operatorname{Tot}(\mathscr{A}) \neq \emptyset($ resp. $\operatorname{Tot}(\mathscr{A})$ est dense dans $H)$ si et seulement si $\operatorname{Tot}\left(\mathscr{A}_{1}\right) \neq \emptyset$ et $\operatorname{Tot}\left(\mathscr{A}_{2}\right) \neq \emptyset\left(\right.$ resp. $\operatorname{Tot}\left(\mathscr{A}_{1}\right)$ est dense dans $H_{1}$ et $\operatorname{Tot}\left(\mathscr{A}_{2}\right)$ dense dans $\left.H_{2}\right)$.

(i) $\Rightarrow$ (iii). Si $\operatorname{Tot}(\mathscr{A}) \neq \emptyset, \mathscr{A}^{\prime}$ est de genre dénombrable et il existe un vecteur $x$ totalisateur pour $\mathscr{A}_{2}$. Puisque $\mathscr{A}_{2}$ est finie, le projecteur [ $\left.\mathscr{A}_{2}^{\prime} x\right]$ est fini, donc $\left[\mathscr{A}_{2} x\right]$ est fini ([4], Chap. III, $\S 2$, Prop. 3). Mais $\left[\mathscr{A}_{2} x\right]=1_{H_{2}}$ donc $\mathscr{A}_{2}^{\prime}$ est finie. D'après [4], Chap. III, $\S 6$, Prop. 3, on a $C_{\mathscr{A}_{2}} \leqq 1$.

(iii) $\Rightarrow$ (ii). Supposons vérifiée la condition (iii). D'après [4], Chap. III, $\S 6$, Prop. 3 et $\S 8$, Cor. 11 du Th. 1 , on a $\operatorname{Tot}(\mathscr{A}) \neq \emptyset$. Soit $x \in \operatorname{Tot}(\mathscr{A})$. D'après la Prop. 1, l'ensemble des $T x$ où $T \in \operatorname{Inv}(\mathscr{A})$ est dense dans $H$. Or si $x \in \operatorname{Tot}(\mathscr{A})$ et $T \in \operatorname{Inv}(\mathscr{A})$, on a $T x \in \operatorname{Tot}(\mathscr{A})$; en effet $\mathscr{A}=\mathscr{A} T$, donc $[\mathscr{A} T x]=[\mathscr{A} x]=1$. Donc $\operatorname{Tot}(\mathscr{A})$ est dense dans $H$ et d'après le Lemme 4 c'est un $G_{\delta}$.

(ii) $\Rightarrow$ (i). C'est évident.

Corollaire 1. Les hypothèses et les notations étant les mêmes que dans la Prop. 2, les 3 conditions suivantes sont équivalentes:

(i) $\operatorname{Tot}(\mathscr{A}) \neq \emptyset$ et $\operatorname{Sep}(\mathscr{A}) \neq \emptyset$.

(ii) $\operatorname{Tot}(\mathscr{A}) \cap \operatorname{Sep}(\mathscr{A})$ est un $G_{\delta}$ dense dans $H$.

(iii) $\mathscr{A}$ et $\mathscr{A}^{\prime}$ sont de genre dénombrable, $\mathscr{A}_{1}^{\prime}$ est proprement infinie, $\mathscr{A}_{2}^{\prime}$ est finie, et $C_{\mathscr{A}_{2}}=1$. 
(i) $\Rightarrow$ (ii). Cela résulte de la Prop. 2 appliquée à $\mathscr{A}$ et $\mathscr{A}^{\prime}$ et du fait que, $H$ étant un espace de Baire, l'intersection de deux $G_{\delta}$ denses est un $G_{\delta}$ dense.

(ii) $\Rightarrow$ (iii). Supposons vérifiée la condition (ii) et soit $\mathscr{A}_{1}=\mathscr{B} \times \mathscr{C}$ avec $\mathscr{B}^{\prime}$ proprement infinie et $\mathscr{C}^{\prime}$ finie. D'après la Prop. 2 appliquée à $\mathscr{A}^{\prime}$, l'algèbre $\mathscr{C}$ est finie, donc $\mathscr{C}=0$ de sorte que $\mathscr{A}_{1}^{\prime}=\mathscr{B}^{\prime}$ est proprement infinie. La Prop. 2 appliquée à $\mathscr{A}$ et $\mathscr{A}^{\prime}$ prouve que $\mathscr{A}_{2}^{\prime}$ est finie et que $C_{\mathscr{A}_{2}}=1$.

(iii) $\Rightarrow$ (i). Cela résulte de la Prop. 2 appliquée à $\mathscr{A}$ et $\mathscr{A}^{\prime}$.

Corollaire 2. Soient $H$ un espace hilbertien et $\left(\mathscr{A}_{1}, \mathscr{A}_{2}, \ldots\right)$ une suite d'algèbres de von Neumann opérant sur H. Les 2 conditions suivantes sont équivalentes:

(i) Pour tout $n$, on a $\operatorname{Tot}\left(\mathscr{A}_{n}\right) \neq \emptyset\left(\right.$ resp. (i') Pour tout $n$, on a $\operatorname{Tot}\left(\mathscr{A}_{n}\right) \neq \emptyset$ et $\left.\operatorname{Sep}\left(\mathscr{A}_{n}\right) \neq \emptyset\right)$.

(ii) $\bigcap_{n \geqq 1} \operatorname{Tot}\left(\mathscr{A}_{n}\right)$ est un $G_{\delta}$ dense dans $H\left(\right.$ resp. (ii') $\bigcap_{n \geqq 1}\left(\operatorname{Tot}\left(\mathscr{A}_{n}\right) \cap \operatorname{Sep}\left(\mathscr{A}_{n}\right)\right)$ est un $G_{\delta}$ dense dans $H$ ).

Cela résulte de la Prop. 2 puisque dans un espace de Baire une intersection dénombrable de $G_{\delta}$ denses est un $G_{\delta}$ dense.

Remarque 1. On retrouve ainsi le Th. 13 de [6].

Remarque 2. Si $\mathscr{A}_{2}=0$ et si $H$ est séparable, on peut démontrer l'implication (iii) $\Rightarrow$ (ii) de la Prop. 2 en observant que $\mathscr{A}$ contient un facteur de type $I_{\infty}$ et en appliquant le cor. $2.11 \mathrm{de}$ [2].

\section{Algèbres d'observables locales}

La partie (ii) de la proposition suivante améliore le Th. 1 de [6].

Proposition 3. Soient $\Omega$ l'ensemble des ouverts bornés de $\boldsymbol{R}^{4}$, et $H$ un espace hilbertien. Soit $\mathcal{O} \mapsto \mathscr{R}(\mathcal{O})$ une application de $\Omega$ dans l'ensemble des algèbres de von Neumann opérant sur $H$. On suppose vérifiées les 2 conditions suivantes:

a) Si $\mathscr{O}_{1} \subset \mathcal{O}_{2}$, on a $\mathscr{R}\left(\mathcal{O}_{1}\right) \subset \mathscr{R}\left(\mathcal{O}_{2}\right)$.

b) Si $\mathcal{O}_{1}$ et $\mathcal{O}_{2}$ sont causalement disjoints, $\mathscr{R}\left(\mathcal{O}_{1}\right)$ et $\mathscr{R}\left(\mathcal{O}_{2}\right)$ sont permutables.

Alors

(i) Siles $\mathscr{R}(\mathcal{O})$ sont proprement infinies et les $\mathscr{R}(\mathcal{O})^{\prime}$ de genre dénombrable, l'ensemble des vecteurs totalisateurs et séparateurs à la fois pour chaque $\mathscr{R}(\mathcal{O})$ est un $G_{\delta}$ dense dans $H$.

(ii) Si chaque $\mathscr{R}(\mathcal{O})$ possède un vecteur totalisateur, il existe un projecteur unique $G$ possédant les propriétés suivantes:

ג) $G$ appartient au centre de toutes les algèbres $\mathscr{R}(\mathcal{O})$; 
B) pour tous $\mathcal{O}, \mathcal{O}_{1} \in \Omega$, on a $\mathscr{R}(\mathcal{O})_{G}=\mathscr{R}\left(\mathcal{O}_{1}\right)_{G}$ et $\mathscr{R}(\mathcal{O})_{G}$ est commutative;

$\gamma)$ pour tout $\mathcal{O} \in \Omega$ l'algèbre $\mathscr{R}(\mathcal{O})_{1-G}$ est proprement infinie.

Démonstration de (i). Soit $\Omega^{\prime}$ l'ensemble dénombrable des boules ouvertes dans $\boldsymbol{R}^{4}$ de rayon rationnel et dont le centre a ses coordonnées rationnelles. Posons $K=\bigcap_{\mathcal{O} \in \Omega^{\prime}} \operatorname{Tot}(\mathscr{R}(\mathcal{O}))$. Pour tout $\mathcal{O} \in \Omega$, il existe $\mathcal{O}_{1} \in \Omega^{\prime}$ tel que $\mathcal{O}_{1} \subset \mathcal{O}$, donc $K=\bigcap_{\mathcal{O} \in \Omega} \operatorname{Tot}(\mathscr{R}(\mathcal{O}))$. Puisque $\mathscr{R}(\mathcal{O})$ est proprement infinie, $\operatorname{Tot}(\mathscr{R}(\mathcal{O}))$ est non vide d'après la Prop. 2. D'après le cor. $2, K$ est un $G_{\delta}$ dense. Mais pour tout $\mathcal{O} \in \Omega$, il existe $\mathcal{O}_{1} \in \Omega^{\prime}$ tel que $\mathcal{O}$ et $\mathcal{O}_{1}$ soient causalement disjoints. Comme $\mathscr{R}\left(\mathcal{O}_{1}\right) \subset \mathscr{R}(\mathcal{O})^{\prime}$, tout élément de $K$ est séparateur pour $\mathscr{R}(\mathcal{O})$. On a donc prouvé (i) et on voit aussi que, sous l'hypothèse (ii), il existe un vecteur totalisateur et séparateur pour chaque $\mathscr{R}(\mathcal{O})$.

Dans la suite, nous nous donnerons un vecteur $x$ totalisateur et séparateur à la fois pour chaque $\mathscr{R}(\mathcal{O})$. Pour dèmontrer (ii), prouvons d'abord les lemmes suivants:

Lemme 5. Soient $\mathcal{O}_{\mathcal{O}_{1}} \in \Omega$ avec $\mathcal{O}_{1} \subset \mathcal{O}$. Soient $P$ un projecteur de $\mathscr{R}(\mathcal{O}) \cap \mathscr{R}\left(\mathcal{O}_{1}\right)^{\prime}, Q$ son support central dans $\mathscr{R}\left(\mathcal{O}_{1}\right)^{\prime}$. Alors $P$ est équivalent à $Q$ relativement à $\mathscr{R}\left(\mathcal{O}_{1}\right)^{\prime}$.

La démonstration est calquée sur celle de [3], Th. 2.5.b

On a $Q=\left[\mathscr{R}\left(\mathcal{O}_{1}\right)^{\prime} P H\right]=\left[\mathscr{R}\left(\mathcal{O}_{1}\right)^{\prime} P \mathscr{R}(\mathcal{O})^{\prime} x\right]=\left[\mathscr{R}\left(\mathcal{O}_{1}\right)^{\prime} \mathscr{R}(\mathcal{O})^{\prime} P x\right]$ $=\left[\mathscr{R}\left(\mathcal{O}_{1}\right)^{\prime} P x\right]$. D'autre part, $Q=\left[Q \mathscr{R}\left(\mathcal{O}_{1}\right)^{\prime} x\right]=\left[\mathscr{R}\left(\mathcal{O}_{1}\right)^{\prime} Q x\right]$. On a donc $\left[\mathscr{R}\left(\mathcal{O}_{1}\right)^{\prime} P x\right]=\left[\mathscr{R}\left(\mathcal{O}_{1}\right)^{\prime} Q x\right]$. D'après [4], Chap. III, $\S 1$, Cor. du Th. 2, on en déduit que, relativement à $\mathscr{R}\left(\mathcal{O}_{1}\right)^{\prime}$, on a $\left[\mathscr{R}\left(\mathcal{O}_{1}\right) P x\right] \sim\left[\mathscr{R}\left(\mathcal{O}_{1}\right) Q x\right]$, c'est-à-dire $P \sim Q$.

Lemme 6. Soient $\mathcal{O} \in \Omega$ et $G$ un projecteur du centre de $\mathscr{R}(\mathcal{O})$ tel que $\mathscr{R}(\mathcal{O})_{G}$ soit finie. Alors:

(i) $\mathscr{R}(\mathcal{O})_{G}$ est commutative.

(ii) Pour tout $\mathcal{O}_{1} \in \Omega$, on a $G \in \mathscr{R}\left(\mathcal{O}_{1}\right) \cap \mathscr{R}\left(\mathcal{O}_{1}\right)^{\prime}$ et $\mathscr{R}\left(\mathcal{O}_{1}\right)_{G}=\mathscr{R}(\mathcal{O})_{G}$.

Supposons d'abord que $\mathcal{O}_{1}>\mathcal{O}$. Le vecteur $G x$ est totalisateur pour $\mathscr{R}(\mathcal{O})_{G}$ donc aussi pour $\mathscr{R}\left(\mathcal{O}_{1}\right)_{G}$. D'autre part $\left[\mathscr{R}\left(\mathcal{O}_{1}\right)^{\prime} G x\right]=\left[G \mathscr{R}\left(\mathcal{O}_{1}\right)^{\prime} x\right]=G$ ce qui montre que $G x$ est totalisateur pour $\mathscr{R}\left(\mathcal{O}_{1}\right)_{G}^{\prime}$ donc séparateur pour $\mathscr{R}\left(\mathcal{O}_{1}\right)_{G}$. Les algèbres $\mathscr{R}\left(\mathcal{O}_{1}\right)_{G}$ et $\mathscr{R}(\mathcal{O})_{G}$ ont donc un vecteur totalisateur et séparateur commun. L'algèbre $\mathscr{R}(\mathcal{O})_{G}$ est finie et $\mathscr{R}(\mathcal{O})_{G} \subset \mathscr{R}\left(\mathcal{O}_{1}\right)_{G}$. D'après [6], Lemme 2, cela entraîne $\mathscr{R}(\mathcal{O})_{G}=\mathscr{R}\left(\mathcal{O}_{1}\right)_{G}$. Soit $G_{1}$ le support central de $G$ dans $\mathscr{R}\left(\mathcal{O}_{1}\right)$. D'après [3] th. 2.5.b on a $G \sim G_{1}$ relativement à $\mathscr{R}\left(\mathcal{O}_{1}\right)$. (Dans [3], Th. 2.5, est faite une hypothèse supplémentaire sur l'algèbre de Von Neumann engendrée par les $\mathscr{R}(\mathcal{O})$. Mais cette hypothèse n'intervient pas dans la démonstration du résultat cité.). Puisque $\mathscr{R}\left(\mathcal{O}_{1}\right)_{G}=\mathscr{R}(\mathcal{O})_{G}$ est une algèbre finie, le projecteur $G$ est fini relativement à $\mathscr{R}\left(\mathcal{O}_{1}\right)$, donc $G_{1}$ est fini et comme $G \leqq G_{1}$ ceci entraîne $G=G_{1}$. Donc $G$ appartient au centre de $\mathscr{R}\left(\mathcal{O}_{1}\right)$. 
Supposons maintenant que $\mathcal{O}_{1} \subset \mathcal{O}$. On a $G \in \mathscr{R}\left(\mathcal{O}_{1}\right)^{\prime}$, on peut donc considérer l'algèbre $\mathscr{R}\left(\mathcal{O}_{1}\right)_{G}$. Le vecteur $G x$, étant séparateur pour $\mathscr{R}(\mathcal{O})_{G}$ est aussi séparateur pour $\mathscr{R}\left(\mathcal{O}_{1}\right)_{G}$. D'autre part $\left[\mathscr{R}\left(\mathcal{O}_{1}\right) G x\right]$ $=\left[G \mathscr{R}\left(\mathcal{O}_{1}\right) x\right]=G$ donc $G x$ est totalisateur pour $\mathscr{R}\left(\mathcal{O}_{1}\right)_{G}$. Le Lemme 2 de [6] permet donc encore de conclure que $\mathscr{R}(\mathcal{O})_{G}=\mathscr{R}\left(\mathcal{O}_{1}\right)_{G}$. L'algèbre $\mathscr{R}\left(\mathcal{O}_{1}\right)_{G}$ est donc finie, et comme elle admet un vecteur totalisateur et séparateur, elle est standard ([4], Chap. III, §1, Th. 5) de sorte que $\mathscr{R}\left(\mathcal{O}_{1}\right)_{G}^{\prime}$ est finie. Le projecteur $G$ est donc un projecteur fini de $\mathscr{R}\left(\mathscr{O}_{1}\right)^{\prime}$. Le Lemme 5 permet de conclure comme précédemment que $G$ est égal à son support central dans $\mathscr{R}\left(\mathcal{O}_{1}\right)^{\prime}$, donc $G$ appartient au centre de $\mathscr{R}\left(\mathcal{O}_{1}\right)^{\prime}$.

Soit maintenant $\mathcal{O}_{1}$ un élément quelconque de $\Omega$. Il existe $\mathcal{O}_{2} \in \Omega$ tel que $\mathcal{O}_{2}>\left(\mathcal{O}_{1} \cup \mathcal{O}\right)$. En appliquant successivement les deux résultats précédents, on en déduit que $G \in \mathscr{R}\left(\mathcal{O}_{1}\right) \cap \mathscr{R}\left(\mathcal{O}_{1}\right)^{\prime}$, et que $\mathscr{R}\left(\mathcal{O}_{1}\right)_{G}=\mathscr{R}(\mathcal{O})_{G}$. La propriété (b) de la Prop. 3 entraîne que $\mathscr{R}(\mathcal{O})_{G}$ est commutative.

Démonstration de (ii).

L'unicité de $G$ est évidente. Prouvons l'existence.

Soit $G$ le plus grand projecteur, dans l'intersection des centres des algèbres $\mathscr{R}(\mathcal{O})$, tel que les $\mathscr{R}(\mathcal{O})_{G}$ soient commutatives. D'après le lemme 6, on a $\mathscr{R}(\mathcal{O})_{G}=\mathscr{R}\left(\mathcal{O}_{1}\right)_{G}$ pour tous $\mathcal{O}, \mathcal{O}_{1} \in \Omega$. Montrons par l'absurde que pour tout $\mathcal{O} \in \Omega$ l'algèbre $\mathscr{R}(\mathcal{O})_{1-G}$ est proprement infinie. Dans le cas contraire, il existerait un projecteur non nul $G_{1} \leqq 1-G$ appartenant au centre de $\mathscr{R}(\mathcal{O})$ tel que $\mathscr{R}(\mathcal{O})_{G_{1}}$ soit finie. D'après le Lemme 6, pour tout $\mathcal{O}_{1} \in \Omega, G_{1}$ appartiendrait au centre de $\mathscr{R}\left(\mathcal{O}_{1}\right)$, et $\mathscr{R}\left(\mathcal{O}_{1}\right)_{G_{1}}$ serait commutative. Cela contredirait la maximalité de $G$.

Remarque 3. Conservons les hypothèses de la Prop. 3. Supposons en outre qu'il existe un vecteur totalisateur et séparateur pour toutes les algèbres $\mathscr{R}(\mathcal{O})$, et que l'algèbre de von Neumann engendrée par les $\mathscr{R}(\mathcal{O})$ soit un facteur, ce qui est le cas dans certaines situations intéressantes (cf. [I] Prop. 2, Cor. I et [3], Th. 2. I). Alors d'après la Prop. 3, ou bien $\operatorname{dim} H=1$, ou bien chaque $\mathscr{R}(\mathcal{O})$ est proprement infinie.

Nous remercions M. Lévy-Nahas pour ses suggestions utiles dans la démonstration de la Prop. 3.

Ajouté en épreuves: Pour des raisonnements analogues à ceux des lemmes 1-3, voir H. Choda, An extremal property of the polar decomposition in von Neumann algebras. Proc. Japan Acad. 46, 341-344 (1970).

\section{Bibliographie}

1. Araki,H.: On the algebra of all local observables. Prog. Theor. Phys. 32 (5), 844-854 (1964).

2. - Woods, E. J.: A classification of factors. Pub. R.I.M.S. Kyoto Univ. Ser. A, Vol. 4, $51-130$ (1968).

4 Commun. math. Phys., Vol. 22 
50 J. Dixmier et $\mathrm{O}$. Maréchal: Vecteurs totalisateurs d'une algèbre de von Neumann

3. Borchers, H.: Lectures in Quantum Field Theory. Princeton, 1965-1966.

4. Dixmier, J.: Les algèbres d'opérateurs dans l'espace Hilbertien $2^{\mathrm{eme}}$ ed. Paris: GauthierVillars 1969.

5. Feldman, J., Kadison, R.V.: The closure of the regular operators in a ring of operators. Proc. Am. Math. Soc. 5, 909-916 (1954).

6. Kadison, R.V.: Remarks on the type of von Neumann algebras of local observables in quantum field theory. J. Math. Phys. 4, 1511-1516 (1963).

\author{
J. Dixmier \\ 64 rue Gay-Lussac \\ Paris (5) \\ France
}

\author{
O. Maréchal \\ Résidence Ronsard \\ 1 rue de la Pléiade \\ 94 L'Hay-les-Roses, France
}

